\title{
SPIDER: A new instrument for fission fragment research at the Los Alamos Neutron Science Center
}

\author{
Fredrik Tovesson ${ }^{1, \mathrm{a}}$, Charles Arnold ${ }^{1}$, Rick Blakeley ${ }^{2}$, Adam Hecht $^{2}$, Alexander Laptev ${ }^{1}$, \\ Drew Mader ${ }^{2}$, Krista Meierbachtol ${ }^{1}$, Lucas Snyder ${ }^{3}$, and Morgan White ${ }^{1}$ \\ ${ }^{1}$ Los Alamos National Laboratory, PO Box 1663, Los Alamos, NM 87545 \\ ${ }^{2}$ University of New Mexico, Dept. of Chemical and Nuclear Engineering, Albuquerque, NM 87131 \\ ${ }^{3}$ Lawrence Livermore National Laboratory, 7000 East Avenue, Livermore, CA 94550
}

\begin{abstract}
The study of fission fragment yields and how they behave as a function of excitation energy provides insight into the process in which they are formed. Fission yields are also important for nuclear applications, as they can be used as a diagnostic tool. A new instrument, SPIDER (Spectrometer for Ion DEtermination in fission Research), is being developed for measuring fission yields as a function of incident neutron energy at the Los Alamos Neutron Science Center. The instrument employs a time-of-flight mass spectrometry method in which the velocity and kinetic energy of the fragments are measured in order to determine their mass. Additionally, by using Bragg peak spectroscopy, the charge of the fragments can be identified. A prototype instrument has been developed and preliminary results indicate that $\sim 1$ mass unit resolution is feasible using this approach. A larger detector array is currently being designed, and will be used at study fission yields from thermal neutron energies up to at least $20 \mathrm{MeV}$.
\end{abstract}

\section{Introduction}

The fission process is a highly complex process, and to this day there are no models that can reliably predict the properties of fragments for various compound system and excitation energies. By measuring the fission fragment kinetic energy, mass and charge for neutron-induced fission of various actinides over a wide incident neutron energy range one can study the systematic trends needed to guide the theoretical modelling and provide data for model validation. Fission yields are also important for nuclear applications: by collecting samples of spent fuel rods from a nuclear reactor and counting the number of atoms of a specific fission product one can infer the burn-up of the fuel.

Fission fragment yields have been measured extensively at certain neutron energies (thermal, fission spectrum, $14 \mathrm{MeV}$ ) for the major actinides, but the energy dependence is generally not well understood. Furthermore, even with the large number of data sets there are still uncertainties of up 5\% for important fission product yields. In order to provide high precision measurements of the fission fragment properties a new instrument is being developed that will be used to study neutron-induced fission at the Los Alamos

\footnotetext{
ae-mail: tovesson@lanl.gov
}

This is an Open Access article distributed under the terms of the Creative Commons Attribution License 2.0, which permits unrestricted use, distribution, and reproduction in any medium, provided the original work is properly cited. 


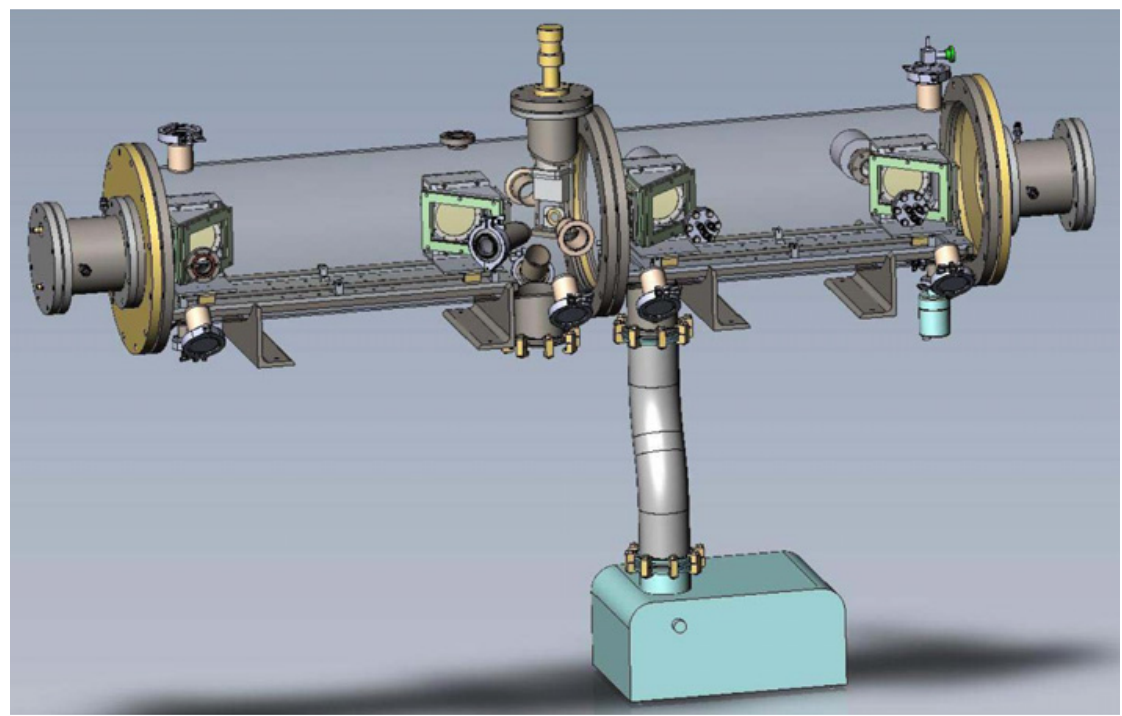

Figure 1. Drawing of the dual-arm version of the SPIDER spectrometer.

Neutron Science Center (LANSCE) [1]. The instrument is based on the 2E-2v method first demonstrated at the ILL in Grenoble, France in the 1980's [2]. The first performance tests of the individual detectors used in the SPIDER instrument are reported on here.

\section{Experiments}

The spectrometer will measure the velocity (v) and kinetic energy (E) of the fragments, which are then used to calculate their mass (in the non-relativistic limit) according to

$$
M=2 \frac{E}{v^{2}} .
$$

Each arm of the spectrometer will have one section measuring the time-of-flight (TOF) of the ions in vacuum, and an ionization chamber that measures the kinetic energy. The goal is to build a spectrometer with close to $1 \mathrm{amu}$ resolution, and this depends on the resolution that can be achieved for energy, time, and flight path length according to

$$
\frac{\Delta M}{M}=\sqrt{\left(\frac{\Delta E}{E}\right)^{2}+\left(2 \frac{\Delta t}{t}\right)^{2}+\left(2 \frac{\Delta l}{l}\right)^{2}},
$$

Where $E$ is the energy, $t$ is the time-of-flight, and $l$ the flight path length. The uncertainty in flight path length depends on two components; the different distances traveled between the two detectors due to variation in particle trajectories and how accurately the distance between the start and stop detectors can be measured. A dual-arm version of the SPIDER spectrometer is currently being assembled, and will be used to measure fission fragment mass distributions for spontaneous fission of ${ }^{252} \mathrm{Cf}$, and thermal neutron-induced fission of ${ }^{235} \mathrm{U}$ and ${ }^{239} \mathrm{Pu}$ at the LANSCE neutron source. A drawing of the spectrometer is shown in Figure 1.

In order to estimate the mass resolution of the spectrometer extensive testing of each of the individual components has been performed to determine the temporal resolution of the time pick-of-detectors used for the TOF section and the energy resolution of the ionization chambers. 


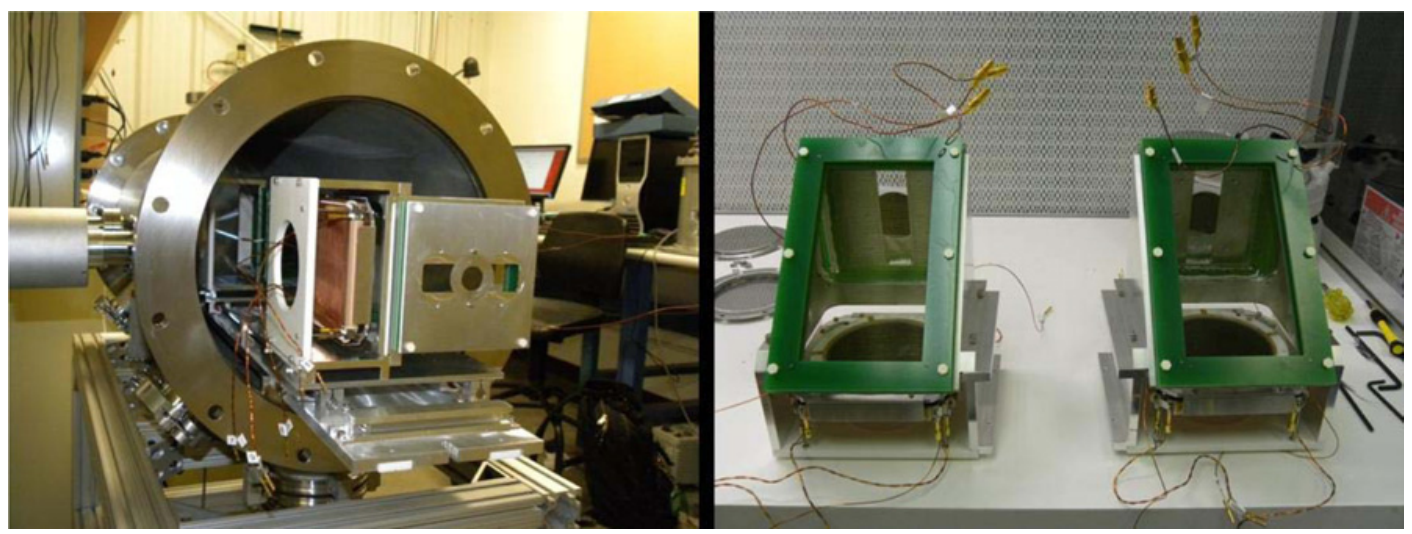

Figure 2. Left: time-of-flight chamber with the detectors partially extracted. Right: a pair of timing detector assemblies.

\subsection{Time-of-flight detector}

The start and stop of the TOF is measured by detecting the secondary electrons emitted as the ions passes through thin $\left(<100 \mathrm{ug} / \mathrm{cm}^{2}\right)$ carbon foils. The electrons are accelerated using an electrostatic potential, and directed towards a micro-channel plate (MCP) using an electrostatic mirror. The MCP provides a fast signal with $2-3 \mathrm{~ns}$ rise time which is sufficiently short to get sub-ns resolution. The electrostatic mirrors are shown in Figure 2, right side.

The timing resolution was investigated by placing the detectors in a vacuum chamber, separated by approximately $50 \mathrm{~cm}$. A Th-229 alpha-source was placed in front of the start detector, and the time-offlight between the start and stop detector was measured. The source has five relatively strong alpha-lines ranging from 4.8 to $8.4 \mathrm{MeV}$. By fitting the width in time of the corresponding peaks the temporal resolution can be extracted.

Another important property of the timing detectors is their efficiency. This was investigated by placing a silicon surface barrier detector behind one of the mirrors. By comparing singles in the silicon detector to coincidences with the mirror, the detection efficiency for alpha particles was determined.

\subsection{Ionization chamber}

Ionization chambers were chosen for their high energy resolution for fission fragments, which is approximately a factor of 2 better than for silicon surface barrier detectors. The axial ionization chamber design is shown in Figure 3. The charged particles enter the ionization chamber through a thin window perpendicular to the anode plane, and stop in the gas filled volume. A Frisch grid shields the anode from the drift of the positive ions produced as the particles stop in the gas, so that only drifting electrons form the signal on the anode. The energy resolution of the chamber was measured using a tri-nuclide alpha source mounted on the inside of the chamber at the position where the thin entrance window will eventually be mounted. An energy spectrum was collected in this configuration, and the widths of the alpha-peaks are used to determine the resolution.

\section{Results}

The measured time-of-flight spectrum for the Th-229 source is shown in Figure 4. The five main alpha lines all have almost identical width, which in this test was $200 \mathrm{ps}$ (FWHM) which corresponds to $140 \mathrm{ps}$ per detector. Based on previous work with these types of detectors we believe that it should be possible 


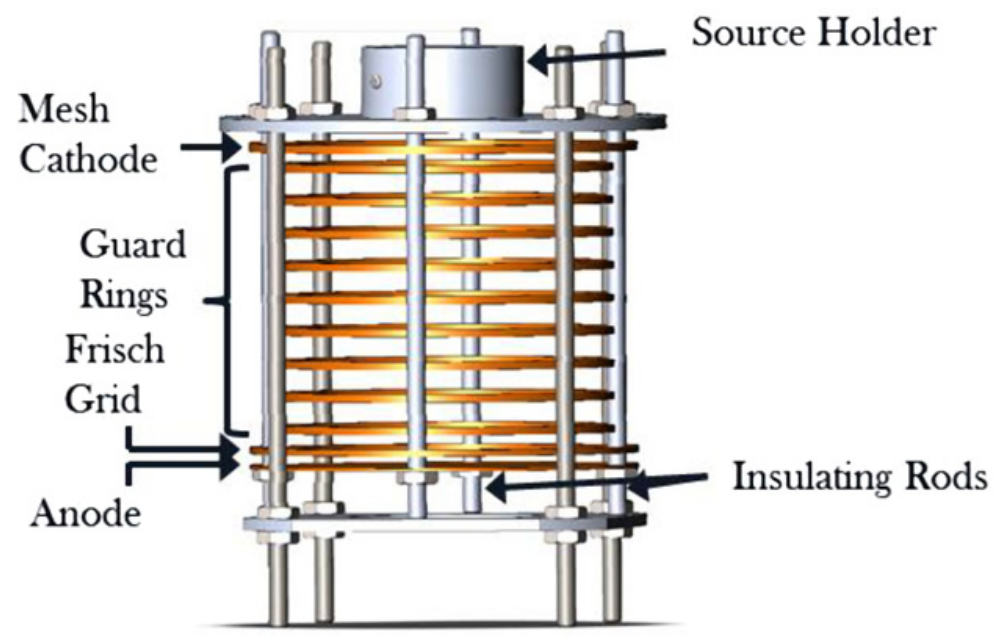

Figure 3. Drawing of the interior assembly of the axial ionization chamber.

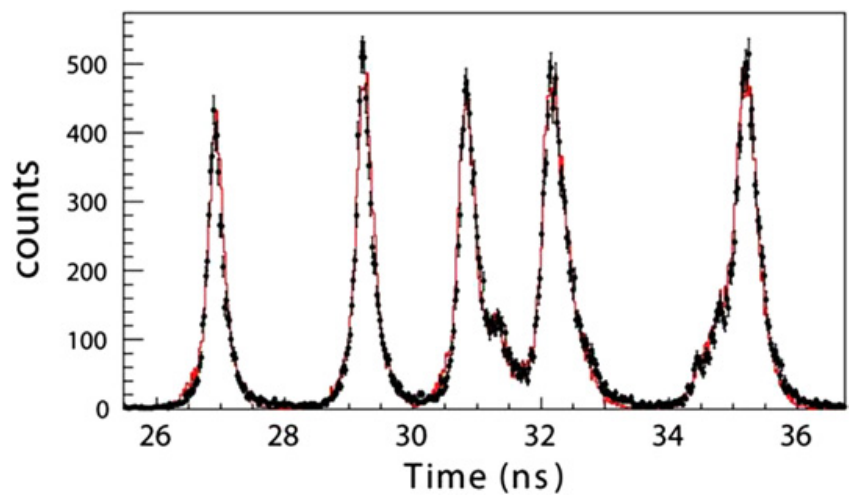

Figure 4. Measured time-of-flight spectrum for the Th-229 source.

to approach 100 ps per detector for fission fragments. Improved analog signal processing might help in reaching this goal.

The efficiency for alpha-particle detection with the timing detector as a function of acceleration potential is shown in Figure 5. At low potentials the electrons have a low probability of reaching the MCP and get detected, and this probability increases steadily with the electric potential up to about $100 \mathrm{~V}$ after which the efficiency reaches a plateau. The 70-80\% efficiency stays constant even as the potential is increased to several $\mathrm{kV}$. While there is no improvement in the efficiency by going above $200 \mathrm{~V}$, the spatial and temporal resolution might be improved by using a high potential and this needs to be studied further.

The energy spectrum of alpha particles measured with the ionization chamber is shown in Figure 6. By measuring the widths of the energy peaks the resolution was determined to be $1.1 \%$. The resolution for fission fragments is typically better than for alpha particles, so sub-1\% resolution is expected.

A fission fragment measurement for ${ }^{239} \mathrm{Pu}$ with the SPIDER instrument was simulated assuming the measure temporal and energy resolution, and a $60 \mathrm{~cm}$ flight path. The expected mass spectrum is shown in Figure 7. The light fragment mass resolution is about $1 \mathrm{amu}$, so individual peaks are observed in this 
Fission 2013

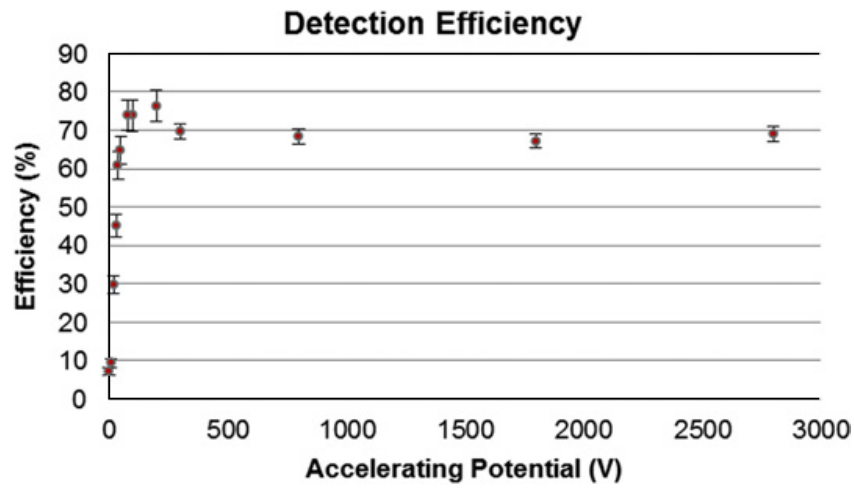

Figure 5. Alpha particle detection efficiency for the time pick-off detectors as a function of acceleration potential.

ionization chamber alpha particle spectrum

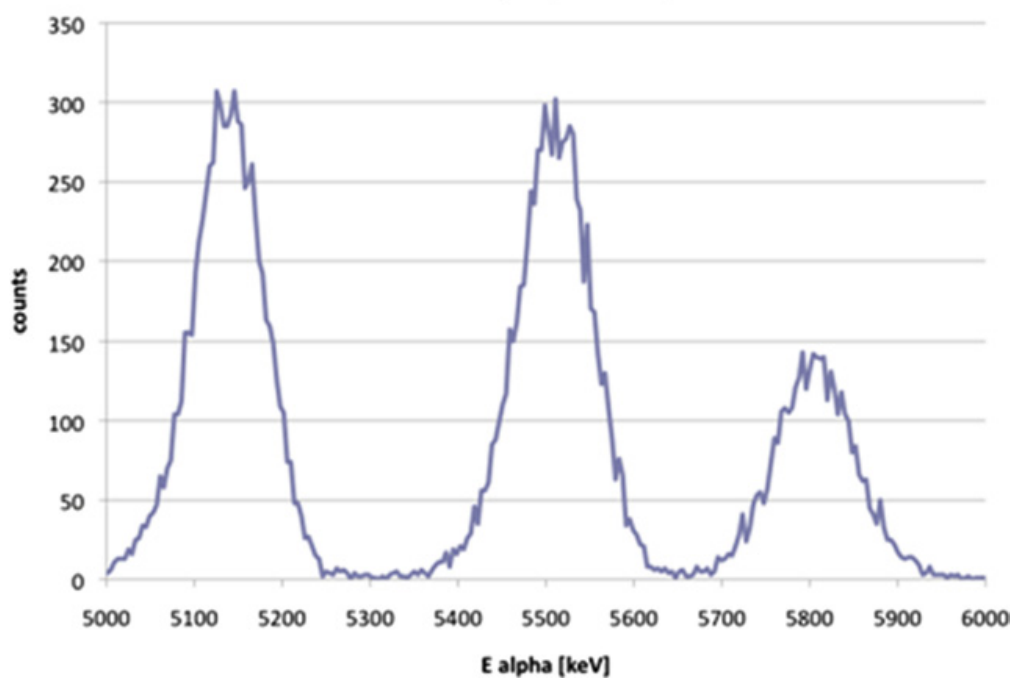

Figure 6. Alpha particle energy spectrum measured with the axial ionization chamber.
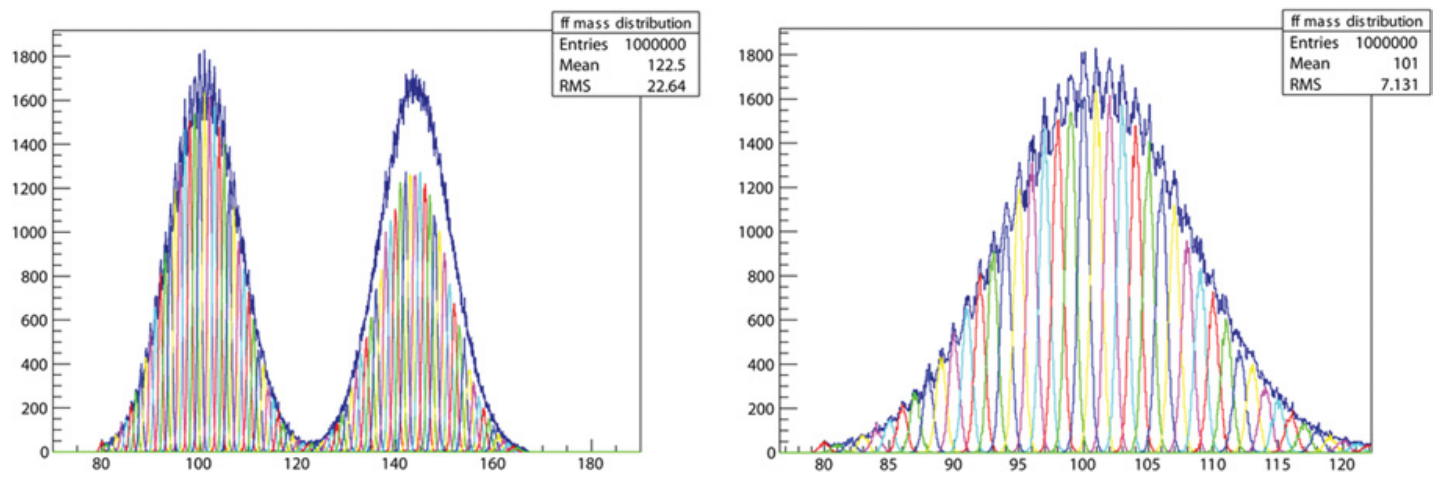

Figure 7. Simulated fission mass distribution from SPIDER. 
region. For the heavy fragments the mass resolution is slightly higher than $1 \mathrm{amu}$, so the individual mass peaks are no longer observed.

\section{Conclusion and outlook}

The performances of the detector components of the SPIDER spectrometer have been investigated. The temporal and energy resolution has been shown to be sufficiently high to meet the goal of about $1 \mathrm{amu}$ resolution for fission fragments. While the detectors themselves meet the performance requirements, there are still challenges to optimize their performance when integrated into the spectrometer. These will be investigated further once the two-arm system is assembled.

After the dual-arm system has been fully tested and used in neutron beam experiments the next step foreseen is to build an array of spectrometer arms to increase the efficiency. This will allow for measurements at fast neutron energies where the fission cross sections of fissile isotopes are significantly lower than at thermal energies. These types of measurements would require an instrument with 8-10 spectrometer arm pairs.

\section{References}

[1] P. W. Lisowski, K. F. Schoenberg, Nucl. Inst. Meth. in Phys. Research A562, 910 (2006)

[2] N. Boucheneb, P. Geltenbort, M. Asghar, G. Barreau, T. P. Doan, F. Gönnenwein, B. Leroux, A. Oed, A. Sicre, Nucl. Phys. A502, 261 (1989) 